\title{
Nonlinear Magnetoelectric Coupling Dynamic Characteristics of Galfenol-piezoelectric Film Sensors
}

\author{
Xiao-Huan Li, ${ }^{1}$ Zhi-Wen Zhu, ${ }^{1}$ Guo-Song Feng, ${ }^{2}$ and Jia $\mathrm{Xu}^{3 *}$ \\ ${ }^{1}$ Department of Mechanics, Tianjin University, \\ 92 Weijin Road, Nankai District, Tianjin 300072, China \\ ${ }^{2}$ Beijing Institute of Spacecraft Environment Enginnnering, Beijing 100094, China \\ ${ }^{3}$ Tianjin Key Laboratory of Nonlinear Dynamics and Control, Tianjin University, \\ 92 Weijin Road, Nankai District, Tianjin 300072, China
}

(Received April 17, 2018; accepted March 20, 2019)

Keywords: Galfenol, piezoelectric ceramics, hysteretic nonlinearity, magnetoelectric response

A type of Galfenol-piezoelectric composite film sensor is proposed in this paper, and its nonlinear magnetoelectric coupling dynamic characteristics are discussed. Nonlinear differential terms are introduced to explain the hysteresis of Galfenol's strain-magnetic-fieldintensity (MFI) curves, and a new Galfenol's model is established. The fitting effect of the Galfenol's model on the experimental data is proved by the partial least-squares regression method. A nonlinear dynamic model for such a sensor subjected to a harmonic magnetic field and a stochastic electromagnetic interference has been developed, and its strongly nonlinear dynamic response is obtained by the developed Lindstedt-Poincare (L-P) method. The results of numerical simulation show that the Galfenol-piezoelectric composite film sensor has complex dynamic characteristics; the stochastic electromagnetic interference plays an important role in the system's dynamic response. The results of this study are helpful for the optimal design and improvement of Galfenol-piezoelectric composite film sensors.

\section{Introduction}

A magnetic sensor is the key component of many electronic devices. The common magnetic sensors are the Hall component and giant magnetostrictive-piezoelectric composite sensor. In some cases, the measured magnetic field is usually submerged in electromagnetic interference (EMI), which leads to the requirements for high-sensitivity sensors. To detect a magnetic field with low intensity, a composite film sensor was proposed. A composite film sensor is made up of two types of films: magnetostrictive and piezoelectric films. The magnetostrictive film has a large surface area to produce a large deformation even in a magnetic field with low intensity, and the piezoelectric film can generate sufficient current based on the deformation induced by the piezoelectric effect. Terfenol-D film, which is a conventional magnetostrictive film, has a high magnetostriction rate. However, Terfenol-D is very brittle and cannot withstand

*Corresponding author: e-mail: xujia@tju.edu.cn https://doi.org/10.18494/SAM.2019.2276 
large load and deformation. Galfenol is a novel giant magnetostrictive material. It has a high magnetostriction rate and good elasticity, making it a promising material for magnetic sensors.

Many researchers have studied magnetostrictive film sensors. Torii et al. proposed a type of Tb-Fe-Co giant magnetostrictive thin film and studied its application to force sensors. ${ }^{(1)}$ Zhou et al. analyzed the transverse, longitudinal, and perpendicular giant magnetoimpedance effects in a compact multiturn meander $\mathrm{NiFe} / \mathrm{Cu} / \mathrm{NiFe}$ trilayer film sensor. ${ }^{(2)}$ NazariNejad et al. designed a giant magnetoimpedance thin film magnetic sensor based on combinations of magnetostrictive and electroactive materials. ${ }^{(3)}$ Zhang and Wang tested a magnetic field sensor using an electrodeposited thin film of giant magnetoresistive $(\mathrm{Cu} / \mathrm{Co})$ multilayers. ${ }^{(4)}$ Luong et al. designed a 3D magnetic field sensor with a single bridge of spin-valve giant magnetoresistance films. ${ }^{(5)}$ Li et al. discussed the integration of a thin film giant magnetoimpedance sensor and a surface acoustic wave transponder. ${ }^{(6)}$ Although many achievements in magnetostrictive film sensors have been reported in previous years, ${ }^{(7-15)}$ theoretical results on the dynamic characteristics of the magnetostrictive film sensors subjected to EMI are few.

In a film sensor, deformation is very large, which causes the strong nonlinear characteristics of the magnetostrictive film. Common analysis methods, such as averaging and multiscale methods, are only adopted for weak nonlinear systems. In this paper, a new Galfenolpiezoelectric composite film sensor is proposed, and its strong dynamic characteristics subjected to harmonic magnetic field and stochastic EMI signals are analyzed by the developed Lindstedt-Poincare (L-P) method. Finally, the effects of the EMI intensity on the system's response are discussed.

\section{Nonlinear Dynamic Model of Galfenol-PVDF Composite Film Sensor}

The structure of a Galfenol-piezoelectric composite film sensor is shown in Fig. 1. The composite film sensor is made up of two types of films: Galfenol and PVDF piezoelectric films. When the sensor is placed in a varying magnetic field, the Galfenol film vibrates, and then the PVDF piezoelectric film is subjected to a varying pressure and generates varying currents. The strain-magnetic-field-intensity (MFI) curves of Galfenol materials are shown in Fig. 2. The curves evidently show hysteretic characteristics. In this study, a Van der Pol hysteretic model was introduced to describe the hysteretic nonlinear characteristics of Galfenol alloy based on the magnetostrictive effect as

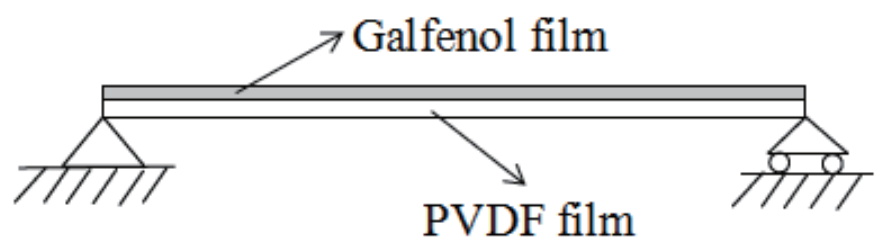

Fig. 1. Structure of Galfenol-PVDF film sensor. 


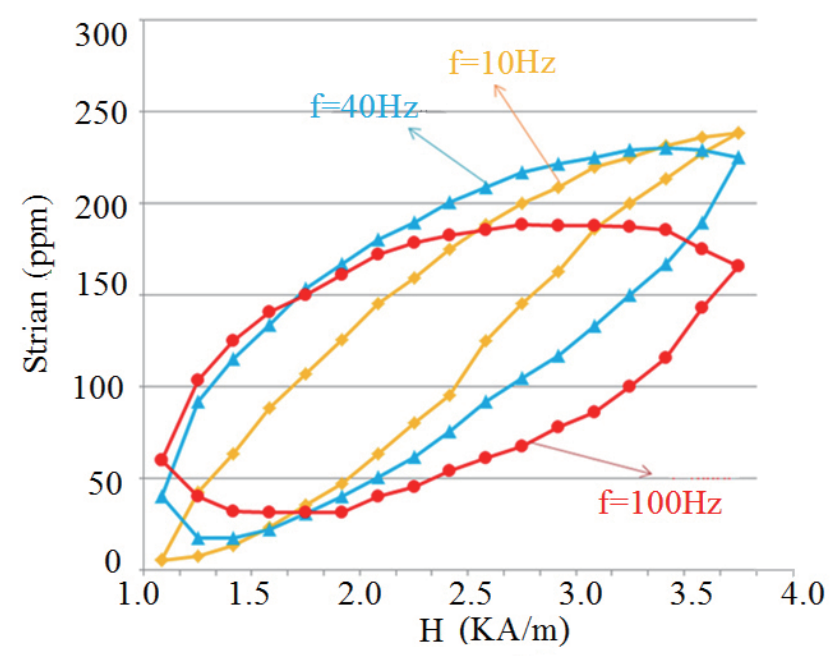

Fig. 2. (Color online) Strain-MFI curves of Galfenol alloy.

$$
\varepsilon=f_{1}(H)+f_{2}(H)=k_{1} H+k_{2} H^{2}+k_{3} H^{3}+\left(k_{4} H+k_{5} H^{2}+k_{6} H^{3}+k_{7} H^{4}\right) \dot{H},
$$

where $\varepsilon$ is the strain, $H$ is the MFI, $k_{i}(i=1-7)$ are the coefficients, $f_{1}(H)=k_{1} H+k_{2} H^{2}+k_{3} H^{3}$ is the skeleton curve of the hysteretic loop, and $f_{2}(H)=\left(k_{4} H+k_{5} H^{2}+k_{6} H^{3}+k_{7} H^{4}\right) \dot{H}$ is the improved Van der Pol term, which describes the difference between the skeleton curve and the hysteretic loop.

The partial least-squares regression method has been used to test the fitting effect of Eq. (1). The results of the principal component analysis based on the experimental data are shown in Fig. 3, and the coefficients are shown in Fig. 4. All the valuable importance (VIP) terms are sufficiently large, indicating that these terms are the principal components of the model.

Now, we can establish the sensor's dynamic model. In this study, the Hamilton variation principle is applied in system modeling as

$$
\delta S=\int \delta\left(T_{1}+T_{2}+M^{K}-U_{1}-U_{2}-M^{P}-W_{d}+W_{O E}\right) d t=0
$$

where $T_{i}$ is the kinetic energy of the two materials, $T_{i}=\frac{1}{2} \int_{0}^{S_{i}} \rho_{i} A_{i}\left(\frac{d q_{i}}{d t}\right)^{2} d s_{i},(i=1,2)$, with 1: Galfenol film and 2: PVDF film. $S_{i}$ is the area of the two films, $\rho_{i}$ is the density, $A_{i}$ is the thickness, and $q_{i}$ is the displacement; $M^{K}$ is the magnetic field energy of the Galfenol film, $M^{K}=\frac{1}{2} \iiint B \cdot K d V$, with $K=K_{0} \sin \Omega t$ being the intensity of the magnetic field; $U_{i}$ is the potential energy of the two materials, $U_{i}=\frac{1}{2} \int_{0}^{S_{i}} \frac{E_{i} A_{i}}{s_{i}} q_{i}^{2} d s_{i}$, $(i=$ $1,2)$, with $E_{i}$ being the elastic modulus; $M^{P}$ is the electric field energy of the PVDF film, 


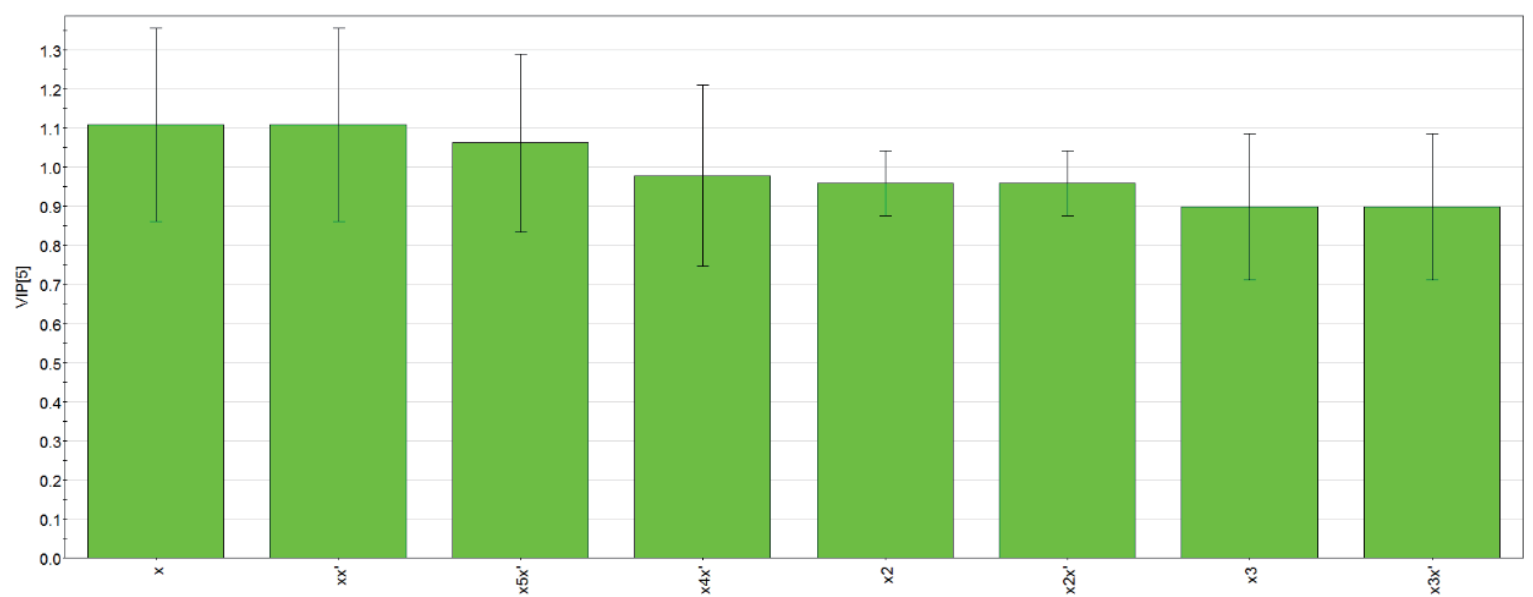

Fig. 3. (Color online) Results of principal component analysis.

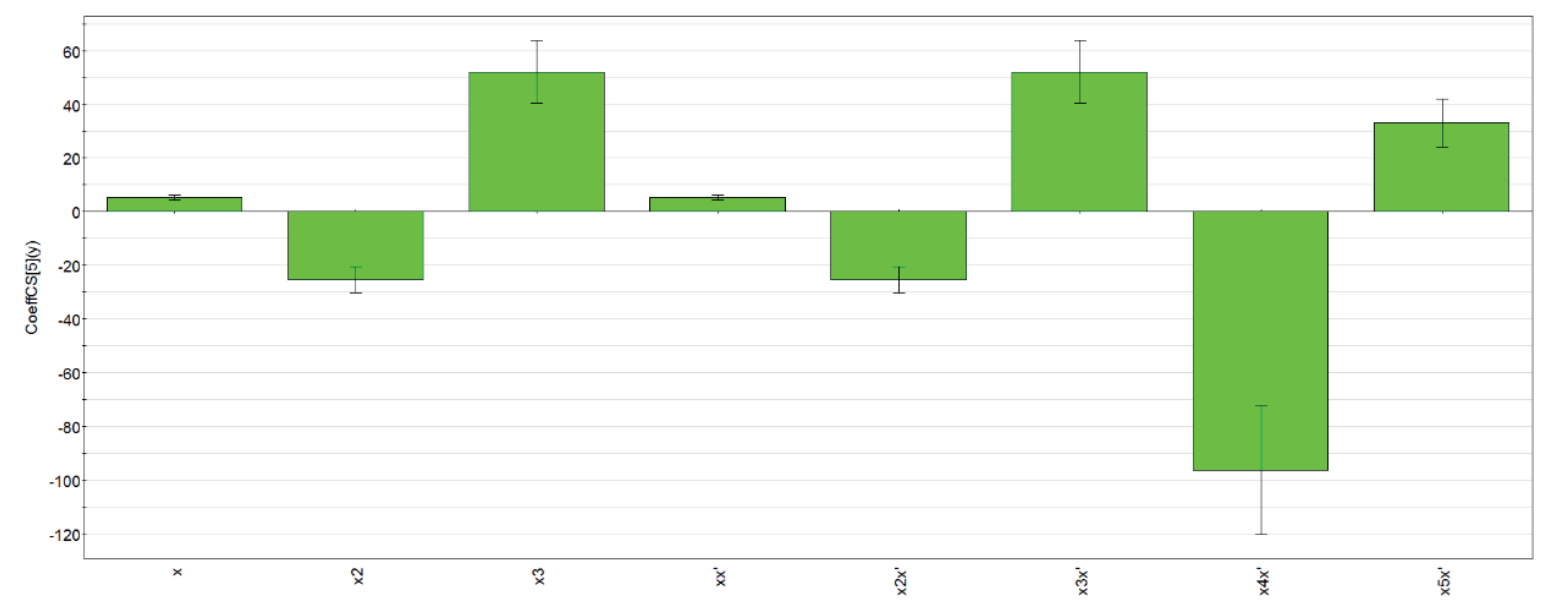

Fig. 4. (Color online) Coefficients.

$M^{P}=\frac{1}{2} \iiint E_{3} \cdot D d V=\frac{A_{1}}{2} \int_{0}^{S_{1}} E_{33} D_{33} d s_{1}$, with $E_{33}=\frac{U}{L_{1}}$ being the electric field intensity, $U$ the output voltage of the PVDF film, and $D_{33}$ the electric displacement. $W_{d}$ is the work performed by the system's damp force, $W_{d}=\int_{0}^{S} c q \frac{\partial q}{\partial t} d s$, with $c$ being the damping coefficient of the structures. $W_{O E}$ is the work performed by in-plane excitation, $W_{O E}=\int_{0}^{L} F d x$. Here, $F$ is the stochastic perturbation caused by EMI, $F=\bar{F} \varsigma(t)$, where $\zeta(t)$ is the normal Gauss white noise.

When the displacement of the middle point of the film is the system's response $q$, we can obtain the dynamic equation of the system's response as

$$
\ddot{q}+\left(2 \eta+c_{4} q^{2}+c_{5} q^{4}\right) \dot{q}+c_{1} q+c_{2} q^{2}+c_{3} q^{3}=\bar{K} \sin \Omega t+e \varsigma(t) .
$$




\section{Strong Dynamic Characteristics of the System}

A large deformation of a film sensor will cause the strong nonlinear characteristics of the film, indicating that Eq. (3) is a strong nonlinear system. Assuming that the coefficient $c_{3}$ of the term $q^{3}$ is the largest among those of all the nonlinear terms and the damping coefficient $\eta$ is small, we let $c_{1}=\omega_{0}^{2}$ and $\varepsilon_{1}=c_{3}$. Thus, we can obtain

$$
\ddot{q}+\omega_{0}^{2} q+\varepsilon_{1}\left[\left(e_{6}+e_{4} q^{2}+e_{5} q^{4}\right) \dot{q}+e_{1} q+e_{2} q^{2}+q^{3}\right]=\bar{K} \sin \Omega t+e \varsigma(t),
$$

where $e_{i}=\frac{c_{i}}{\varepsilon_{1}},(i=1,2,4,5)$ and $e_{6}=\frac{2 \eta}{\varepsilon_{1}}$. Obviously, $e_{i}<1$ since $\varepsilon_{1}=c_{3}$ and $c_{3}$ is the largest among those of all the nonlinear terms. However, $\varepsilon_{1}=c_{3}$ may still be large, and we cannot use a nonlinear method directly. This means that we must make $\varepsilon_{1}$ small. In this paper, the developed L-P method is applied to solve the strong nonlinear equation to obtain the natural frequency of the strong nonlinear system.

Let

$$
\begin{gathered}
\tau=\omega_{0} t \\
\omega^{2}=\omega_{0}^{2}+\omega_{1} \varepsilon_{1}+\omega_{2} \varepsilon_{1}^{2}+\ldots, \\
\alpha=\frac{\omega_{1}^{2} \varepsilon_{1}}{\omega_{0}^{2}+\omega_{1}^{2} \varepsilon_{1}} \\
q=q_{0}+\alpha q_{1}+\alpha^{2} q_{2} .
\end{gathered}
$$

Thus,

$$
\begin{gathered}
\omega^{2}=\omega_{0}^{2}+\alpha \frac{\omega_{0}^{2}}{\omega_{1}}, \\
\varepsilon_{1}=\alpha \frac{\omega_{0}^{2}}{\omega_{1}^{2}}+o\left(\alpha^{2}\right) .
\end{gathered}
$$

Using the above transformation, we change $\varepsilon_{1}$ to a small number $\alpha$, and obtain the following equations in accordance with the coefficients of $\alpha^{0}$ and $\alpha^{1}$ : 


$$
\left\{\begin{array}{l}
\frac{d^{2} q_{0}}{d \tau^{2}}+q_{0}=0 \\
\frac{d^{2} q_{1}}{d \tau^{2}}+q_{1}=-\frac{1}{\omega_{1}} \frac{d^{2} q_{0}}{d \tau^{2}}-\frac{1}{\omega_{1}^{2}}\left(e_{6} \omega \frac{d q_{0}}{d \tau}+e_{4} \omega q_{0}^{2} \frac{d q_{0}}{d \tau}+e_{5} \omega q_{0}^{4} \frac{d q_{0}}{d \tau}+e_{2} q_{0}^{2}+q_{0}^{3}\right)
\end{array}\right.
$$

Equation (11) is a weak nonlinear system, and we can obtain the natural frequency of the system as

$$
\omega=\omega_{0} \sqrt{1+\frac{3}{4} c_{3}^{2} a^{2}},
$$

where $a$ is determined by the equation

$$
e_{6}+e_{4} a^{2}+e_{5} a^{4}=0
$$

From the above natural frequency $\omega$, we obtain the drift coefficient $m(H)$ and diffusion coefficient $\bar{\sigma}(H)$ of Eq. (4) as

$$
\begin{gathered}
m(H)=\left(\frac{D e^{2}}{\omega^{2}}-2 \eta\right) H-\frac{c_{4}}{2 \omega^{2}} H^{2}-\frac{c_{5}}{4 \omega^{2}} H^{4}, \\
\sigma^{2}(H)=\frac{D e^{2}}{\omega^{2}} H^{2} .
\end{gathered}
$$

The averaged Fokker-Planck-Kolmogorov (FPK) equation of Eq. (4) is

$$
\frac{\partial f}{\partial t}=-\frac{\partial}{\partial H}[m(H) f]+\frac{1}{2} \frac{\partial^{2}\left[\sigma^{2}(H) f\right]}{\partial H^{2}},
$$

where $f$ is the stationary probability density (SPD) of the system's response. Thus,

$$
f(H)=\bar{A} H^{-\frac{4 \eta \omega^{2}}{D e^{2}}} \exp \left[-\frac{c_{4}}{D e^{2}} H-\frac{c_{5}}{2 D e^{2} \omega^{2}} H^{2}\right] .
$$

The numerical results of the system response are presented in Fig. 5, where $c_{1}=800, c_{2}=$ $0.2, c_{3}=1, c_{4}=-0.3, c_{5}=0.4, D=0.5$, and $\eta=0.015$. From Eq. (17) and Fig. 5, we obtain the following results: 


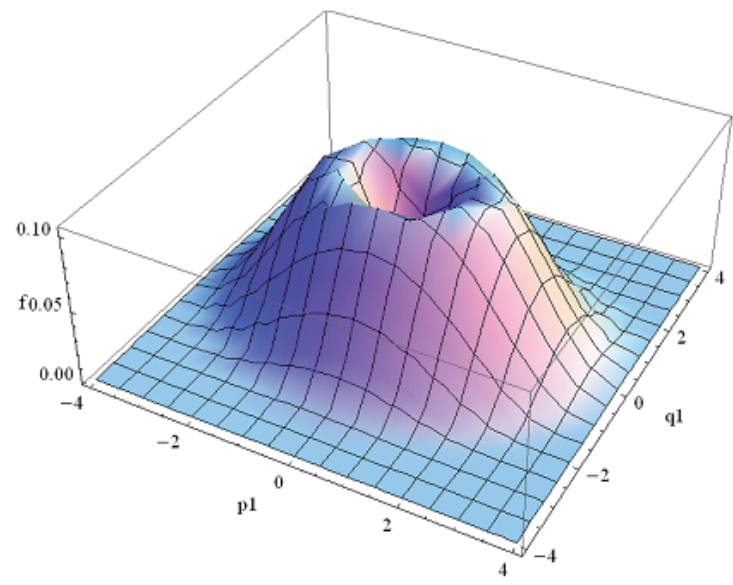

(a)

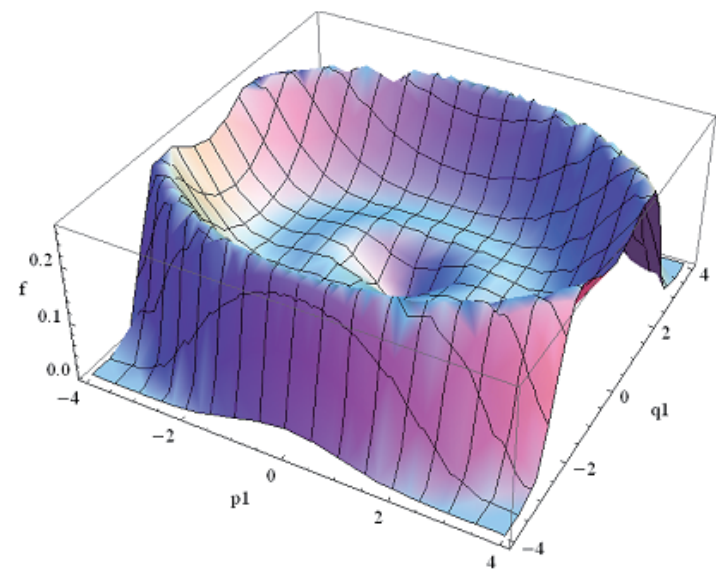

(c)

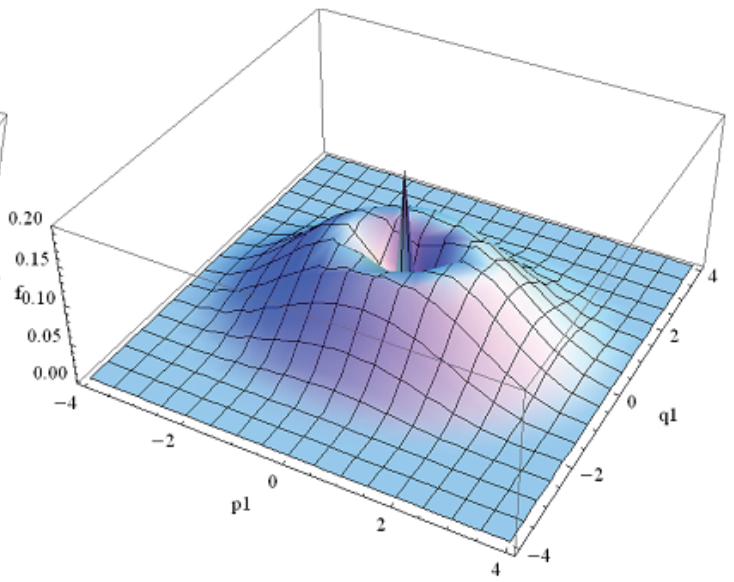

(b)

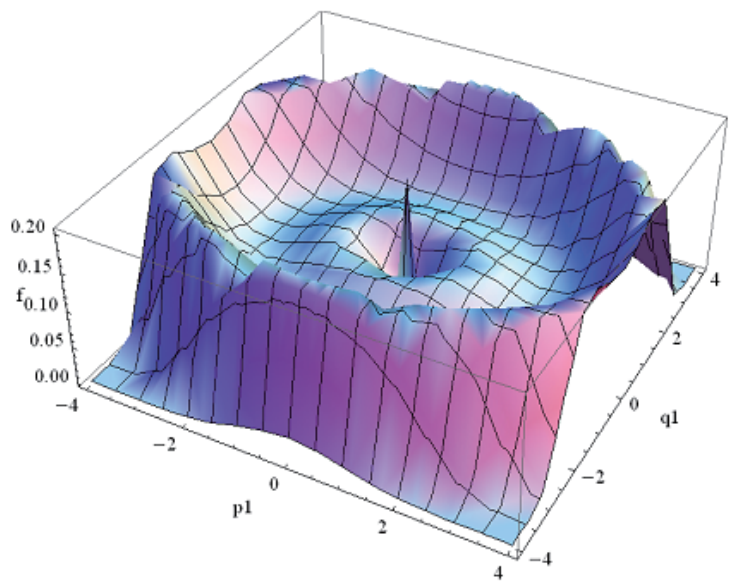

(d)

Fig. 5. (Color online) Probability density of the system's response when $K=0.5$ and (a) $e=0$, (b) $e=0.2$, (c) $e=0.5$, and (d) $e=1$.

A loop is formed in the SPD map when $K=0.5$ and $e=0$, as shown in Fig. 5(a). This means that the system's motion and the output voltage are both periodic when the film sensor is only in a harmonic magnetic field.

A peak and a loop are formed in the SPD map when $K=0.5$ and $e=0.2$, as shown in Fig. 5(b). This means that the system's motion can be of two types: a periodic motion with large amplitude and a vibration near the balance point $(0,0)$. The type of motion the system should have is determined by the initial conditions.

In the variation process of the system's parameters, the system's response can jump from the balance point to periodic orbits under external excitation, inducing the stochastic Hopf bifurcation of the system.

Two loops are formed in the SPD map when $K=0.5$ and $e=0.5$, as shown in Fig. 5(c). This means that the system's motion can be of two types: a periodic motion with large amplitude and a periodic motion with small amplitude. The type of motion the system should have is determined by the initial conditions. The system's response can jump from one periodic orbit to 
the other under external excitation when the system's parameters change, inducing the periodic orbit bifurcation of the system.

Two loops and a peak are formed in the SPD map when $K=0.5$ and $e=1$, as shown in Fig. 5(d). This means that the system's motion can be of three types: a periodic motion with large amplitude, a periodic motion with small amplitude, and a vibration near the balance point $(0,0)$. The type of motion the system should have is determined by the initial conditions. The system's response can jump from one periodic orbit to the other or from the balance point to periodic orbits when the system's parameters change, and the periodic orbit or Hopf bifurcation of the system occurs accordingly.

\section{Conclusions}

A type of Galfenol-piezoelectric composite film sensor is proposed in this paper, and its nonlinear magnetoelectric coupling dynamic characteristics are discussed. Nonlinear differential terms are introduced to explain the hysteresis of the Galfenol's strain-MFI curves, and a new Galfenol's model is established. The fitting effect of the Galfenol's model on the experimental data is proved by the partial least-squares regression method. A nonlinear dynamic model for such a sensor subjected to a harmonic magnetic field and a stochastic EMI has been developed, and its strongly nonlinear dynamic response is obtained by the developed L-P method. The results of numerical simulation show that the Galfenol-piezoelectric composite film sensor has complex dynamic characteristics; the stochastic EMI plays an important role in the system's dynamic response; when the EMI intensity is sufficiently low, the system's motion is periodic, and the sensor can work efficiently; when the EMI intensity is sufficiently high, the system has complex dynamic characteristics, and the periodic orbit or Hopf bifurcation occurs accordingly. The results of this study are helpful for the optimal design and improvement of Galfenol-piezoelectric composite film sensors.

\section{Acknowledgments}

The authors gratefully acknowledge the support of the Natural Science Foundation of China (NSFC) through Grant Nos. 11872266 and 51875396, the Chinese Aviation Science Foundation through Grant No. 2016ZA48001, the Tianjin Research Program of Application Foundation and Advanced Technology through Grant No. 16JCYBJC18800, and Cast-Bisee 511 through Grant No. CAST-BISEE2017-006.

\section{References}

1 Y. Torii, H. Wakiwaka, and T. Kiyomiya: J. Magn. Magn. Mater. 290 (2005) 861.

2 Z. M. Zhou, Y. Zhou, and L. Chen: Meas. Sci. Technol. 22 (2011) 035202.

3 S. NazariNejad, A. A. Fomani, and R. Mansour: IEEE Trans. Magn. 49 (2013) 3874.

4 H. Zhang and W. Wang: Microsyst. Technol. 9 (2003) 436.

5 V. S. Luong, J. T. Jeng, and B. L. Lai: IEEE Trans. Magn. 51 (2015) 4004504.

6 B. D. Li, N. P. Salem, and I. Giouroudi: J. Appl. Phys. 111 (2012) 07E514.

7 J. Xu, R. Z. Li, and Z. W. Zhu: Sens. Mater. 27 (2015) 633. 
8 D. A. Fillippov: Phys. Solid State 47 (2005) 1080.

9 P. Li, Y. M. Wen, and L. X. Bian: Appl. Phys. Lett. 90 (2007) 022503.

10 V. M. Petrov, G. Srinivasan, and M. I. Bichurin: Phys. Rev. B 75 (2007) 224407.

11 C. S. Lee, J. Joo, and S. Han: Appl. Phys. Lett. 85 (2004) 1841.

12 Z. Y. Jia, W. Liu, and Y. S. Zhang: Sens. Actuators, A 128 (2006) 158.

13 J. Xu, Y. X. Kong, and H. L. Wang: Sens. Mater. 28 (2016) 631.

14 S. Masuda, Y. Matsumura, and Y. Nishi: J. Jpn. Ins. Met. 70 (2006) 166.

15 N. Tiercelin, V. Preobrazhensky, and P. Pernod: J. Magn. Magn. Mater. 210 (2000) 302.

\section{About the Authors}

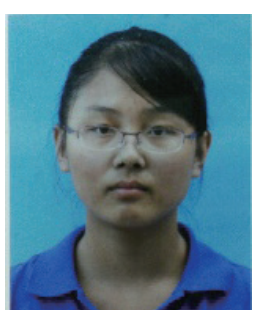

Xiao-Huan Li received her B.S. degree in Engineering Mechanics from Tianjin University, China, in 2018. She is now studying at Tianjin University as a Ph.D. student. Her current research interests focus on the nonlinear dynamics and bifurcation of smart structures.

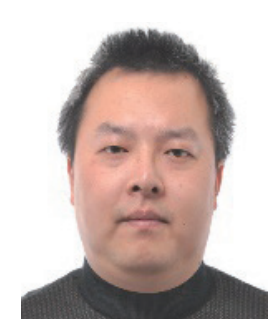

Zhi-Wen Zhu received his B.S. degree from Shanghai Jiaotong University, China, in 1996 and his M.S. and Ph.D. degrees from Tianjin University, China, in 2000 and 2003, respectively. From 2003 to 2005, he was a postdoctoral researcher at Tianjin University, China. In 2005, he was an assistant professor at Tianjin University and became an associate professor at Tianjin University in 2010. His research interests are in the nonlinear dynamics and control of smart structures.

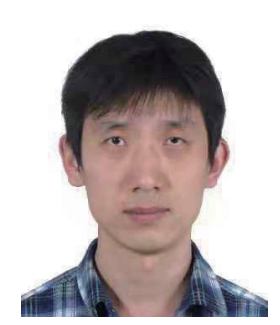

Guo-Song Feng received his B.S. degree from Beijing University of Aeronautics and Astronautics, China. Since 2009, he has been working at Beijing Institute of Spacecraft Environment Engineering. His research interests are in the environmental simulation and test of spacecraft dynamics.

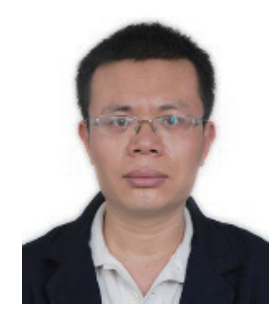

Jia Xu received his B.S., M.S., and Ph.D. degrees from Tianjin University, China, in 2003, 2005, and 2008, respectively. Since 2008, he has been an assistant professor at Tianjin University. His research interests are in the stochastic dynamics analysis and control of nonlinear systems. 